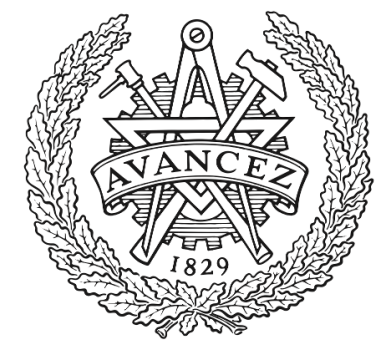

CHALMERS

UNIVERSITY OF TECHNOLOGY

\title{
The NASA 2D wall-mounted hump simulated using DDES-SA with the G3D::Flow solver
}

Downloaded from: https://research.chalmers.se, 2023-04-26 07:36 UTC

Citation for the original published paper (version of record):

Siggeirsson, E., Andersson, N. (2019). The NASA 2D wall-mounted hump simulated using DDES-SA with the G3D::Flow solver. AIAA Scitech 2019 Forum.

http://dx.doi.org/10.2514/6.2019-0083

N.B. When citing this work, cite the original published paper. 


\title{
The NASA 2D wall-mounted hump simulated using DDES-SA with the G3D::Flow solver
}

\author{
Siggeirsson, Elias M. V.* and Andersson, Niklas ${ }^{\dagger}$ \\ Chalmers University of Technology, SE-41296 Gothenburg, Sweden
}

\begin{abstract}
With the interest in simulating complex geometries using the Delayed Detached Eddy Simulation (DDES) model, an initial step should be taken in verifying the methodologies needed. The DDES model is used with a newly proposed modification to the sub-grid lengthscale, the shear-layer-adaptive length-scale, to improve the transition from RANS to LES. The well-known 2D NASA wall-mounted hump test case is simulated. RANS simulations are performed to verify a correct implementation of the turbulence model developed by Spalart and Allmaras (SA). The SA model is important as it will serve as the underlying sub-grid-scale model for the DDES. Furthermore, RANS was used in an initial grid study. Two simulations are performed using the DDES model, where the difference lies in the number of cells and the grid topology. The results show an extended steady shear-layer in the separated region, delaying the transition from RANS to LES, where the cause is suggested to be insufficient grid resolution in the focus region. This influences the prediction of the re-attachment location and the velocity profiles downstream of the hump. However, one of the transient simulations improves the predictions of the re-attachment location and downstream velocity profiles. The other transient simulation is, however, not capable of improving the RANS results due to the delayed breakdown of two-dimensional coherent structures generated at the separation location. The results from the two DDES simulations indicate that the grid-resolution near the separation point needs refinement for a faster transition from RANS to LES. Using an explicit CFD solver for transient simulations of wall-bounded flow configurations, special treatment is needed to make the time-step requirements restricted by flow physics, rather than the numerical stability-limit. To achieve this, the dual-time stepping method has been implemented in to the in-house CFD solver, G3D::Flow. When using the dual-time stepping method, in combination with residual smoothing and low-speed preconditioning, a speed-up of approximately 50 is achieved.
\end{abstract}

\section{Nomenclature}

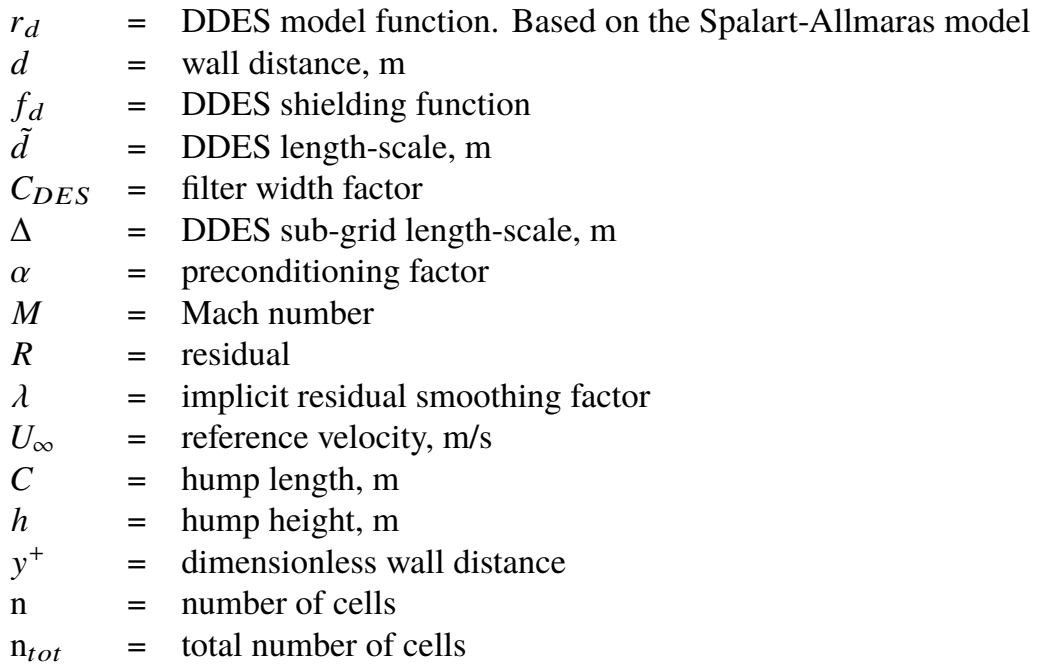

*PhD Student, Division of Fluid Dynamics, Mechanics and Maritime Sciences

${ }^{\dagger}$ Associate Professor, Division of Fluid Dynamics, Mechanics and Maritime Sciences 


$\begin{array}{ll}v_{t} & =\text { eddy viscosity, } \mathrm{m}^{2} / \mathrm{s} \\ \mathbf{\Omega} & =\text { vorticity rate tensor, } 1 / \mathrm{s} \\ \mathbf{S}, \hat{\mathbf{S}} & =\text { strain rate tensor, } 1 / \mathrm{s} \\ C_{f} & =\text { skin-friction coefficient } \\ C_{p} & =\text { pressure coefficient } \\ \Delta t & =\text { time-step, } \mathrm{s} \\ x, y, z & =\text { cartesian coordinates } \\ i, j & =\text { cells } \mathrm{i} \text { and } \mathrm{j} \\ n, m & =\text { vertices } \mathrm{n} \text { and } \mathrm{m} \\ r e f & =\text { reference state. In this case based on the inlet conditions } \\ n b & =\text { neighbouring cells }\end{array}$

\section{Introduction}

Computational resources are increasing every year, making higher fidelity models a practical option for industrial applications. With a great interest in applying the in-house Computational Fluid Dynamics (CFD) solver, G3D::Flow, to industrial graded applications, verifications are needed.

The G3D::Flow solver is an explicit solver for compressible flows, causing the time-step being limited by numerical stability instead of physics. However, the difference between the numerical and physical time-step requirements can be several orders of magnitude, especially when simulating grids with $y^{+}<1$ for the first wall-normal cell. Considering the extremely short time-step requirements, transient simulations, where steady-state acceleration techniques are not possible, are very time-consuming and therefore not practical for grids with high number of cells. To overcome this problem, the explicit solver can be combined with the dual-time stepping method [1, 2]. In the dual-time stepping method a pseudo time-step is introduced. It is a fictitious time-step used to propagate a solution from one physical time-step to the next one, with a separate iterative process, referred to as inner-iterations. Each inner-iterative process is then treated as a steady-state problem. The advantage of this method is that within the pseudo time-stepping routine the common steady-state acceleration techniques can be used (such as local-time stepping, preconditioning and implicit residual smoothing).

With the intention to apply higher-fidelity models to the configuration presented in [3], a verification of the required methodologies is needed. Simulations are performed on a well-established verification case, often referred to as the 2D NASA wall-mounted hump [4], where extensive experimental and CFD work has been conducted. Using such a well-defined test case will allow for the evaluation of the large time-steps possible due to the application of the dual-time stepping method and the performance of the high-fidelity model, the Delayed Detached Eddy Simulation (DDES) model [5].

The DDES model is based on the original Detached Eddy Simulation (DES) model, which was introduced by Spalart et al. [6] as a hybrid method between Large Eddy Simulation (LES) and the one equation Spalart-Allmaras (SA) Reynolds-Averaged Navier-Stokes (RANS) turbulence model [7, 8]. Initially the model was only presented for a 2D application but extended to 3D by Shur et al. [9]. In that study, the performance of the DES model gave a promising result which lead to a wide usage. It was however shown that for grids with stream-wise grid spacing of similar size as the boundary layer, a premature switching from RANS to LES could occur [5]. This was the motivation for the new and improved DES, the DDES model. In the DDES model the switching between RANS and LES is no longer governed only by the grid density but also the flow itself. This has greatly improved the application of the model as less time can be spent in fulfilling the strict grid requirements of the DES model.

Originally, a couple of DDES model coefficients were calibrated using a flat-plate boundary layer but recent studies have shown that some modifications might be needed to achieve good shielding for specific problems, especially geometries were strong stream-wise gradients are present. Ashton [10] presented a study for a three element airfoil, where a problem regarding the shielding function was encountered. The conclusion was that even though the DDES model was applied, the stream-wise and span-wise grid sizes were to fine. The fine grid, in addition to strong pressure gradients over curved surfaces, caused the shielding function to break down and allowed for LES mode inside the boundary layer. Probst et al. [11] encountered a similar problem for a simple airfoil. In their opinion a coarser grid was not an option as high resolution in the stream-wise direction was required to capture the adverse pressure gradient. Instead, they suggested a slight modification to one of the coefficients. This modification was analysed, using the G3D::Flow solver, by Siggeirsson et al. [12]. There, an airfoil was simulated with the standard and modified versions, where improvements were observed using the modification. Therefore, the modified model is considered for this study. 
In this paper, the 2D NASA wall-mounted hump is simulated using both the SA and DDES-SA turbulence models, where the SA model is used as the underlying Sub-Grid-Scale (SGS) model for the DDES. First the G3D::Flow solver is presented along with important settings. Then, the case setup is presented in terms of geometry, boundary conditions and grid topology, where six different grids are evaluated. The results are then compared between the G3D::Flow solver using RANS and DDES as well as RANS results from an external CFD solver and experimental data.

\section{Numerics}

The finite-volume in-house CFD solver for compressible flow, called G3D::Flow雨 is used. The solver is based on a family of codes developed by Eriksson [13]. The solver is developed and maintained at the Division of Fluid Dynamics at Chalmers University of Technology. For steady-state calculations, it uses three-stage Runge-Kutta time marching method with a third-order accurate upwind-biased scheme for all convective terms and a second-order accurate compact centred scheme for all diffusive terms. For the combination of transient and DDES, a second-order, backward differencing, three-stage Runge-Kutta, dual-time marching method is used. The diffusive terms are discretised in the same way as for the steady-state simulations whereas for the convective terms, a third-order accurate low-dissipation upwind-biased scheme [14] is used. Pure central differencing has been proven to be unstable and result in unphysical flow features [15, 16], which is the reason for applying a scheme with small amount of up-winding. For all simulations, a first-order upwind scheme is used for the SA-viscosity to achieve higher stability during the simulations. For more details on the numerical scheme, see Eriksson [13] or Andersson et al. [17].

The DDES-SA model is based on the SA one-equation turbulence model, where a shear-layer-adaptive (SLA) sub-grid length-scale is used. The main motivation for using a hybrid model is that it combines the advantages of the LES capability in resolving the large scales of the transient flow-features and the RANS in simulating the attached boundary layer with relatively low computational cost. The adaptive length-scale has shown to be superior to the standard $\Delta=\max (\Delta x, \Delta y, \Delta z)$ length-scale, where it has a more rapid transition from RANS to LES [18, 19].

\section{A. DDES}

The functionality of the DDES model is based on the boundary layer shielding function $f_{d}$,

$$
f_{d}=1-\tanh \left[\left(8 r_{d}\right)^{3}\right]
$$

The $f_{d}$ function is designed to be 0 in the RANS region but 1 in the LES region, where $r_{d} \ll 1$.

The length-scale, proposed by the original DES model was only based on the wall distance and a grid spacing $\Delta$,

$$
\tilde{d}_{D E S}=\min \left(d, C_{D E S} \Delta\right)
$$

This however, can result in a grid induced separation, therefore a modified length-scale, the DDES length-scale, was introduced as

$$
\tilde{d}_{D D E S}=d-f_{d} \max \left(0, d-C_{D E S} \Delta\right)
$$

When $f_{d}=0$, the model behaves like a RANS model while $f_{d}=1$ results in the original DES model shown in Eq. (2). Shur et al.[9] compared the SA as a SGS model to the Smagorisnky SGS model and experimental data. There, a good agreement was achieved using the filtering factor $C_{D E S}=0.65$ for the DDES model, simulating isotropic turbulence.

A modified shielding function is implemented into G3D::Flow [12], where a stronger shielding is achieved.

$$
f_{d, 16}=1-\tanh \left[\left(16 r_{d}\right)^{3}\right]
$$

Furthermore, it has been shown that when using the SA model as a base model for DDES, the $f_{t 2}$ should be excluded in the equations. It has the tendency to cause the boundary layer to stay laminar over the geometry on finer grids [20].

\section{B. Adaptive length-scale}

For industrial focused grids, the spacing in one direction tends to be larger than the others (for example the z-direction for the hump case and the radial direction for an axial turbomachinery), resulting in large length-scale definition when

*Solver home page: https://nikander.github.io/g3dflow/ 
using the original equation, $\Delta_{\max }=\max [\Delta x, \Delta y, \Delta z]$. To overcome this problem, a new definition of the length-scale, $\Delta_{S L A}$, has been introduced by Shur et al. [18],

$$
\Delta_{S L A}=\tilde{\Delta}_{\omega} F_{K H}(<V T M>)
$$

with

$$
\tilde{\Delta}_{\omega}=\frac{1}{\sqrt{3}} \max _{\mathrm{n}, \mathrm{m}=1,8}\left|\left(\mathbf{I}_{n}-\mathbf{I}_{m}\right)\right|
$$

where $\mathbf{I}_{n}=\mathbf{n}_{\omega} \times \mathbf{r}_{n} . \mathbf{n}_{\omega}$ is the unit vector in the same direction as vorticity vector and $\mathbf{r}_{n}$ is the radius of the $\mathrm{n}^{\text {th }}$ vertex of a specific cell. To recover the behaviour of the original $\Delta_{\max }$ when calculating $3 \mathrm{D}$ turbulence on cubic cells, the cross-product is divided with $\sqrt{3}$.

When considering a free shear-layer flow in the xy plane, with relatively large spacing in the z-direction, the original $\Delta_{\max }$ is dominated by the spacing in the z-direction. The $\tilde{\Delta}_{\omega}$ length-scale, however, is of the order of max $[\Delta x, \Delta y]$ (when the vorticity vector is aligned with the z-direction). Using $\Delta_{\max }$, is still insufficient in rapidly changing from RANS to LES and therefore further modifications are needed to drive the SGS viscosity down in the initial shear-layer region.

To identifying the quasi-2D flow regions, in where the transition from RANS to LES should be accelerated, the dynamic parameter Vortex Tilting Measure $(V T M)$ is introduced

$$
V T M=\frac{\sqrt{6}|(\hat{\mathbf{S}} \cdot \omega) \times \omega|}{\omega^{2} \sqrt{3 \operatorname{tr}\left(\hat{\mathbf{S}}^{2}\right)-\left(\operatorname{tr}(\hat{\mathbf{S}})^{2}\right)}}
$$

where $\operatorname{tr}()$ denotes the trace. VTM takes the value of 0.0 for quasi-2D flows and 1.0 for 3D turbulent flows. This allows for the construction of Eq. (5). To achieve a function with the right behaviour, an empirical function is constructed

$$
F_{K H}(<V T M>)=\max \left[F_{K H}^{\min }, \min \left[F_{K H}^{\max }, F_{K H}^{\min }+\frac{F_{K H}^{\max }-F_{K H}^{\min }}{a_{2}-a_{1}}\left(<V T M>-a_{1}\right)\right]\right]
$$

where $F_{K H}^{\max }=1.0$. The empirical parameters are specified as $F_{K H}^{\min }=0.1, a_{1}=0.15$ and $a_{2}=0.3$. The angle brackets denote averaging of VTM over the current cells and its closest neighbours.

The quantity $<V T M>$ strongly oscillates in the inviscid far field regions since there, the vorticity vector fluctuates around zero. To prevent this instability, the averaged VTM value is modified from the original definition [19]

$$
<V T M>=<V T M>\cdot \max \left(1, \frac{0.2 v}{\max \left[\left(v_{t}-v_{t, \infty}\right), 10^{-6} v_{t, \infty}\right]}\right)
$$

To make sure that $\langle V T M>=1.0$, when the boundary layer is simulated in RANS mode, the shielding function from the DDES model is used as

$$
F_{K H}^{\lim }=\left\{\begin{array}{l}
1.0 \text { if } f_{d}<(1-\varepsilon) \\
F_{K H} \text { if } f_{d} \geq(1-\varepsilon)
\end{array}\right.
$$

where $\varepsilon=0.01$, is an empirical constant.

Combining everything, a final length-scale, which is inserted into Eq. (3), is obtained

$$
\Delta_{S L A}=\tilde{\Delta}_{\omega} F_{K H}^{\lim }\left(\langle V T M\rangle \cdot \max \left(1, \frac{0.2 v}{\max \left[\left(v_{t}-v_{t, \infty}\right), 10^{-6} v_{t, \infty}\right]}\right)\right)
$$

\section{Acceleration techniques}

The preconditioning method used is built on the work done by Eriksson [21], and is based on an isentropic pressure-under relaxation to slow down the acoustic wave speeds to the same order of magnitude as that of the convection-diffusion. There exists an optimum value $\alpha$ for the preconditioning, where

$$
\alpha \approx \frac{u^{2}+v^{2}+w^{2}}{c^{2}}=M^{2}
$$


To make the condition more stable in close proximity to walls, separation regions and for transonic flows, a modification to the optimum $\alpha$ value is applied

$$
\alpha=\min \left[\max \left(M^{2}, \alpha_{\min }\right), 1.0\right]
$$

where the max condition makes sure that no preconditioning is applied to sonic and super-sonic flows. The $\alpha_{\min }$ is set to prevent a singular matrix when $M=0$, where $\alpha_{\text {min }}$ can range from $1-5 \times M_{\text {ref }}$, dependant on how difficult it is to achieve a converged solution [22], where $M_{\text {ref }}$ is a reference number often taken at the inlet of the domain.

To take further advantages of the dual-time stepping and achieve faster convergence for the inner iterations, an Implicit-Residual Smoothing (IRSM) is applied in the pseudo time domain. This stabilizes the solution, allowing for up to double critical CFL number of the Runge-Kutta scheme. The IRSM used is based the smoother presented in [23] with couple of modification where the smoothing is done simultaneously in all directions for each cell and cell stretching is accounted for. The smoothened residuals is obtained with

$$
\bar{R}_{i}=\frac{R_{i}+\lambda \sum_{n b} \bar{R}_{j}}{1+\lambda \sum_{n b} 1}
$$

where $\bar{R}$ represents the smoothened residuals, $R$ the unsmoothed residuals, nb the neighbouring cells and $\lambda$ the smoothening factor

$$
\lambda \leq \frac{1}{4}\left[\left(\frac{C F L}{C F L^{*}}\right)^{2}-1\right]
$$

where $C F L^{*}$ is the stability limit of the unsmoothed Runge-Kutta scheme (for the three stage Runge-Kutta $C F L^{*}=1.2$ ).

\section{Setup}

A flow over a hump, presented in Fig. 1, is simulated to establish the solvers capabilities when performing higher-fidelity simulations.

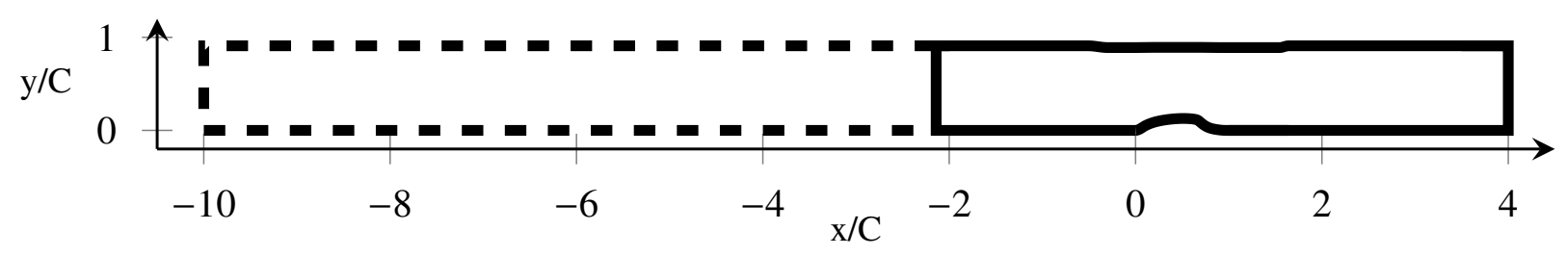

Fig. 1 Computational domain for the 2D Hump.

The hump has a Reynolds number of 936,000, based on a bulk velocity $U_{\infty}=34.5 \mathrm{~m} / \mathrm{s}\left(M_{\infty}=0.1\right)$ and the hump's length $C=0.42 m$ where the height of the hump is $h=0.128 C$. The stagnation properties, pressure and temperature, are specified at the inlet whereas the static pressure is set at the outlet. The lower wall, i.e. the hump, is defined as a viscous wall, whereas the upper wall is defined as an inviscid wall. The drop in the upper wall accounts for side-walls present in the experiments, lowering the cross-sectional area. To ensure that the flow is well developed before reaching the hump, the downstream boundary for the RANS simulations extends to $x / C=-10$. To save computational resources when performing the higher fidelity transient simulations, developed inlet-profiles for density, pressure, SA-viscosity and velocity, are extracted at $x / C=-2.14$ from the previous RANS simulations. The solid lines in Fig. 1 show the hump domain used in the simulations and the dashed line represents the extended inlet used in the RANS simulations. Figure 2 presents the extracted velocity-profile from a RANS simulation, compared to experimental data at $x / C=-2.14$. To better visualise the data, the height of the domain is normalised with the hump's length, whereas the velocity-profile is normalized with the bulk velocity. As seen in the figure the CFD solver is capable of reproducing the experimental velocity-profile upstream of the hump. Additionally, the imported velocity-profile for the DDES is shown, where insignificant difference is noticed through the boundary layer. 


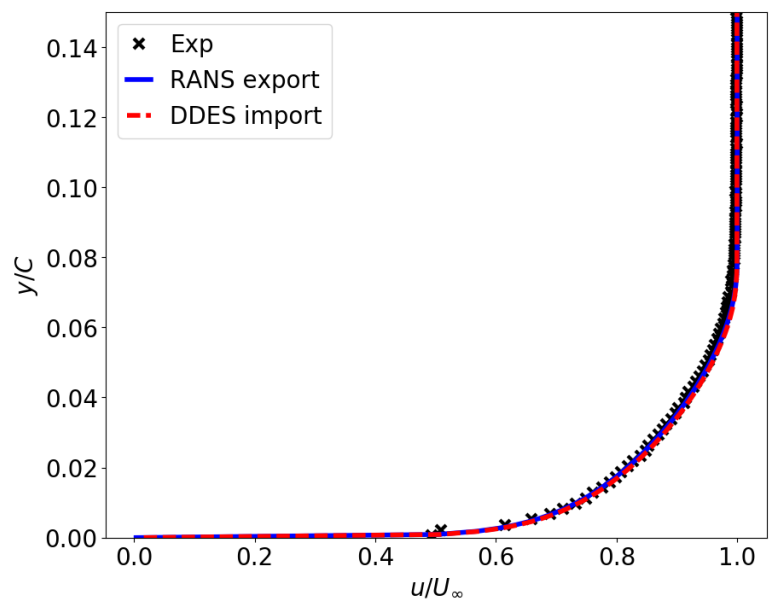

Fig. 2 Comparison of the velocity profile from the CFD simulations and the experiments at $x / C=-2.14$.

To verify the grid topology and further increase confidence in the implementation of the underlying SA turbulence model, 2D steady-state RANS simulations are performed. Previously the SA implementation has been verified with couple of well-known verification cases [24]. The RANS grid topology represents a grid where the cell sizes, in the $\mathrm{x}$-direction downstream of the estimated re-attachment location, are increased exponentially towards the outlet of the domain. This is done to lower the number of cells needed with satisfactory grid resolution, with increased number of cells in the estimated separation region. However, for the DDES2 grid, the region downstream of the hump is the focus region of the simulation $(0.65<x / C<1.5)$. There, a sufficient grid-resolution, capable of resolving the large-scale turbulence, is needed. Furthermore, RANS simulations are performed on each of the DDES grids for comparison.

Four RANS and two DDES grids are generated for grid dependency analysis. In Table 1 the statistics for each grid is presented where number of nodes in $x$ - and $y$-directions ( $n_{x}$ and $n_{y}$, respectively) are shown for the all grids as well as number of nodes in the $z$-direction $\left(n_{z}\right)$ for the DDES grids. The span-wise width of the domain is $z / C=0.2$ with 60 cells for the DDES1 and DDES2 grids. Additionally, the total number of cells are presented in the table, where substantially higher number of cells are obtained for the two DDES grids due to the addition of the z-direction. The DDES1 grid is generated by extruding the RANS2 grid in the z-direction, excluding the extended inlet, whereas the DDES2 grid is generated with a new topology, designed to have slightly larger cells in the separation region but more uniform towards the outlet of the domain.

\section{Table 1 Grid information for the hump.}

\begin{tabular}{|c|r|r|r|r|}
\hline Grid & $n_{x}$ & $n_{y}$ & $n_{z}$ & $n_{\text {tot }}$ \\
\hline RANS1 & 123 & 45 & - & $0.006 \mathrm{M}$ \\
\hline RANS2 & 249 & 90 & - & $0.02 \mathrm{M}$ \\
\hline RANS3 & 501 & 180 & - & $0.09 \mathrm{M}$ \\
\hline RANS4 & 1005 & 360 & - & $0.36 \mathrm{M}$ \\
\hline DDES1 & 236 & 90 & 60 & $1.27 \mathrm{M}$ \\
\hline DDES2 & 474 & 180 & 60 & $5.12 \mathrm{M}$ \\
\hline
\end{tabular}

Figure 3a presents the RANS3 grid whereas in Fig. 3b, a 2D representation of the DDES2 grid is shown. There, the difference between the two topologies can be seen, where the DDES2 topology has higher number of cells in the region downstream of the hump, resulting in more uniform cell sizes. The RANS topology takes advantage of high expansion ratio in the stream-wise direction, to cluster cells between the expected separation region. The DDES1 grid is based on the same topology as used for the RANS grids, except that the short inlet with specified inlet profiles is used. This is done to cluster cells between the separation and re-attachment points, resulting in smaller cells in the initial part of the focus region. Furthermore, the upstream region for the RANS grids (visible in Fig. 1) is not presented but there, a substantial amount of cells are needed. The time-step in the transient simulations for the two DDES grids 
is equal to $4 \cdot 10^{-3} C / U_{\infty}$ to ensure that the $\mathrm{CFL}<1$ in the focus region. To achieve statistically steady-state for the transient simulations, three through-flows are simulated before averaging the results during five through-flows to obtain the time-averaged results.

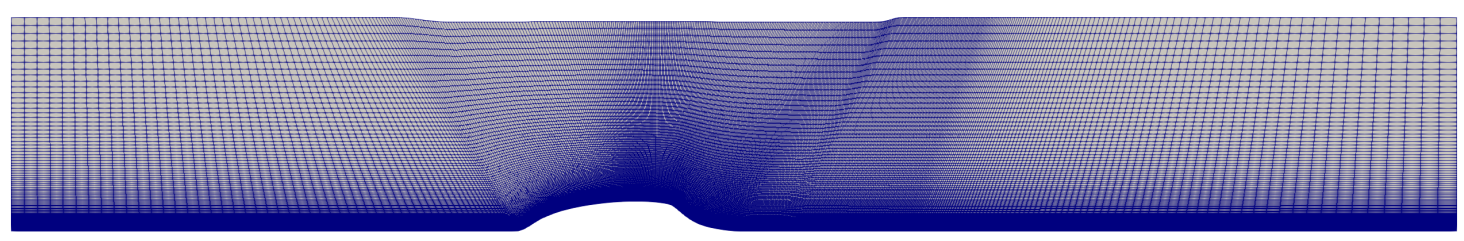

(a) The RANS3 grid. The extended inlet has been removed for better visualisation.

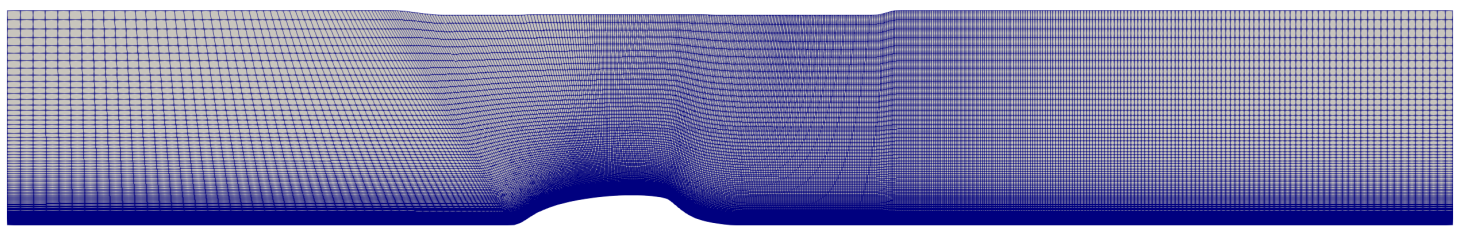

(b) The DDES2 grid with a short inlet.

Fig. 3 2D representation of the grid topologies.

The transient simulations use the dual-time stepping method with maximum of 50 inner iterations. Additionally, a low-speed preconditioning on the inner-iterations is specified with $\alpha_{\min }=6 M_{r e f}^{2}=0.06$ and the IRSM of the inner-iterations is limited to two smoothing operations with $\lambda=0.5$ (with $\approx 1.3 \times C F L^{*}$ to be conservative). For the steady-state simulations, the same specifications are used for the preconditioning, where $2 \times C F L^{*}$ is obtained using the IRSM. This results in substantially faster convergence for the steady-state simulations.

\section{Result}

The steady-state 2D RANS results are evaluated by comparing the results to the CFL3D solver [4] to verify the performance of the underlying RANS model. Additionally, RANS simulations are performed on a 2D representation of the DDES grids to verify the setup, mainly the effect of the specified inlet profiles, and the grid topology. With a verified RANS model and grid topology, the unsteady simulations will be compared to the steady results and experimental data [25] in terms of re-attachment point, skin-friction and pressure coefficients, and the velocity profiles downstream of the hump. Furthermore, the transition from RANS to LES from the DDES results will be analysed in respect of vorticity magnitude and turbulent viscosity.

\section{A. RANS}

In Fig. 4 the predicted re-attachment point downstream of the hump is shown as a function of the number of cells in the stream-wise direction. The comparison is made between the different grids presented in Table 1. Considering the RANS grid topology, the re-attachment point location is converging, with the difference between the last two grids being under $1 \%$. For the DDES grid topology, a similar behaviour is observed for the two simulations using the DDES grids, where the difference is suspected to be due to the specified inlet velocity profile and the slightly different grid topology.

The skin-friction coefficient, as a function of x-location, is shown in Fig. 5a. There, the results obtained with G3D::Flow on the RANS3 and DDES2 grids, are compared to the CFL3D solver as well as experimental data. The RANS3 result are the best in reproducing the experimental data, where main difference from the other CFD simulations, compared to the experiments, is at the top of the hump. The DDES3 results over-estimates the skin-friction at the top of the hump, compared to the experimental data as well as the other CFD results. This might be caused by the specified inlet profiles, where a substantial difference is present at $x / H=-0.5$ between the DDES2's skin-friction and the other two CFD results. Furthermore, all three CFD results show a similar behaviour downstream of the hump, where the re-attachment location is approximately at $x / C=1.24$ and is over-predicted compared to the experimental data, where 


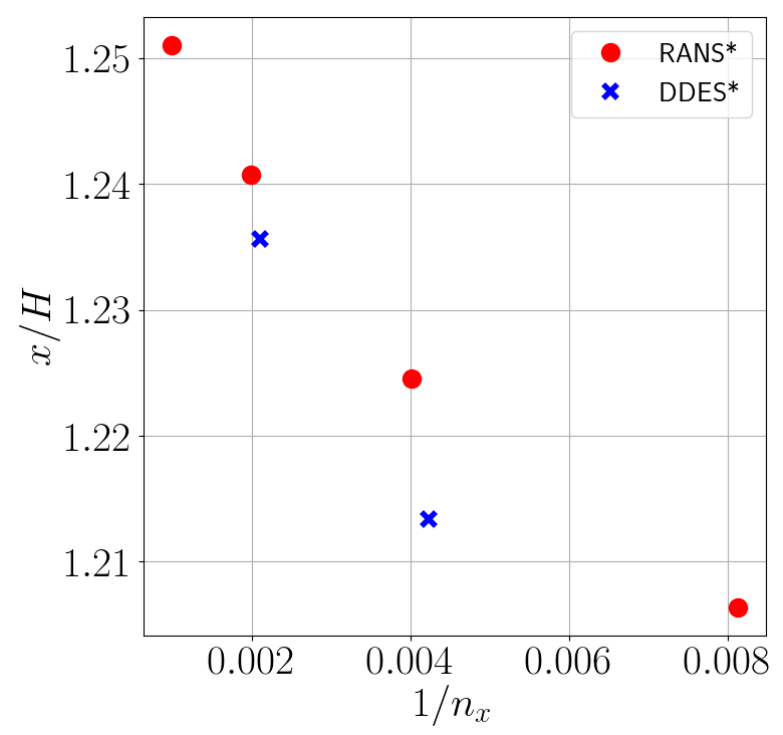

Fig. 4 Re-attachment location in a grid-dependency study.

$x / H \approx 1.1$ for the experimental re-attachment location.

Figure $5 \mathrm{~b}$ shows the pressure coefficient as a function of the $\mathrm{x}$-location for the RANS3 and DDES2 simulations. The results are compared to the CFL3D results as well as experimental data. For a better comparison, the pressure coefficient for the G3D::Flow simulations is shifted to match the CFL3D reference point, upstream of the hump, at $x / C=-2.14$ (where same procedure was done for the CFL3D data). The CFD simulations are capable of reproducing the pressure coefficient up to the separation point, where a clear undershoot is observed. All CFD simulations show a similar behaviour, where the simulation on the DDES2 grid diverges from the other two CFD simulations downstream of the hump. This could, as for the skin-friction coefficient, be a result of a slightly different inlet-profiles and grid topology.

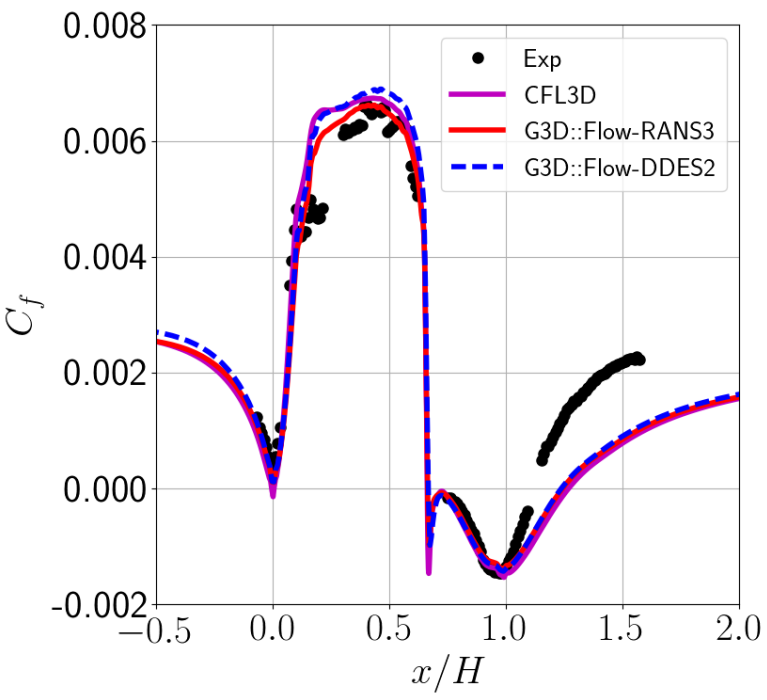

(a) Skin-friction coefficient.

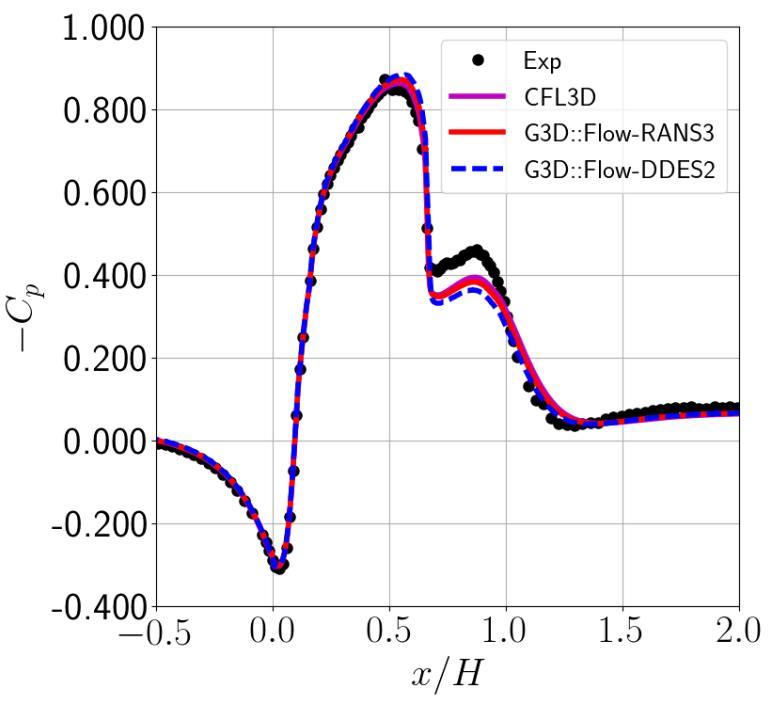

(b) Pressure coefficient.

Fig. 5 Comparison between steady-state results and experimental data along the lower wall.

To further analyse the performance of the RANS simulations, the velocity profiles downstream of the hump are presented in Fig. 6. There, normalized velocities at seven different $x$-locations are compared between the DDES2 
results and the experimental data. Insignificant difference was observed between the CFD results and therefore only one simulation is considered. Overall, the CFD results fail to capture the behaviour near the wall, where for the last three profiles $(x / C=1.1,1.2,1.3)$ the difference is much greater than for the upstream ones. This further presents the delayed re-attachment of the flow when comparing the CFD simulations to the experimental data.

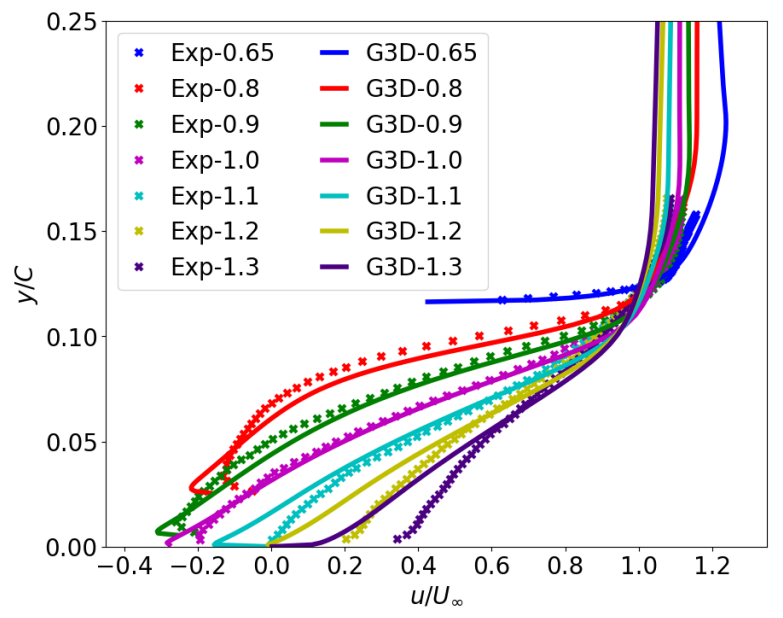

Fig. 6 RANS velocity profiles downstream of the hump at $x / C=\{0.65,0.8,0.9,1.0,1.1,1.2,1.3\}$.

\section{B. DDES}

In Fig.7a, the time-averaged, transient skin-friction coefficient, obtained from the DDES1 and DDES2 results is compared with experimental data as well as the steady-state simulations on the DDES2 grid (SS-DDES2). Considering the DDES1 results, where the DDES1 grid is based on the RANS grid topology with cells clustered around the separation region, the skin-friction is over-predicted over the hump. This is however the same behaviour as seen when simulating the hump using the SA turbulence model, which was expected as the attached boundary layer over the hump is solved using the underlying RANS model. Downstream of the hump, the simulation is not capable of predicting the behaviour of the skin-friction from the experiments, where a delay in decreasing skin-friction is observed and the flow re-attaches at $x / C \approx 1.14$. Compared to the SS-DDES2 RANS simulation, the DDES1 results are only able to improve the re-attachment location while it fails to capture the exact flow-behaviour in the separated region. Considering the DDES2 result, the same behaviour is observed as for DDES1 grid but the transition delay is more severe, causing the predicted re-attachment location to be further downstream (at a similar location as predicted by the RANS simulations).

Figure $7 \mathrm{~b}$ compares the pressure coefficient obtained from the same results as in Fig. $7 \mathrm{a}$. For the pressure coefficient, similar trend is observed for the DDES1 and steady-state DDES2 results, where the transient results on the DDES2 grid are not capable of capturing the slope, downstream of the hump.

Figure 8 shows the instantaneous, eddy viscosity contours at $z=z_{\max } / 2$ for the two transient simulations. For visibility, the contours are presented in a logarithmic scale. As can be seen, the viscosity for the DDES1 results is much higher for stream-wise positions downstream of $x / C=0.9$ compared to the DDES2 result. There is, however, a sign of earlier instability in the DDES1 shear-layer. To further investigate this behaviour the normalized vorticity magnitude is presented in Fig. 9. There, the results from the DDES1 and DDES2 grids are presented. When considering the DDES1 result, the decay in 3D turbulence towards the outlet of the domain is due to the exponentially growing cells. Furthermore, a delayed transition to resolved 3D turbulence is observed, through the presence of a steady shear-layer downstream of the separation point. This coincides with the skin-friction coefficient results seen in Fig. 7a, where the behaviour of the skin-friction was not captured downstream of the separation point. For the results obtained using the DDES2 grid, the same behaviour is seen, with a more severe delay in transition to resolved 3D turbulence, resulting in a longer steady shear-layer which results in the worse prediction of the skin-friction coefficient presented in Fig. 7a. However, the DDES2 grid is capable of sustain a substantially higher level of 3D turbulence, when it has formed, compared to the DDES1 grid, as seen in Fig. 9 . This is, again, due to the exponential growth in cell sizes towards the outlet in the DDES1 grid. 


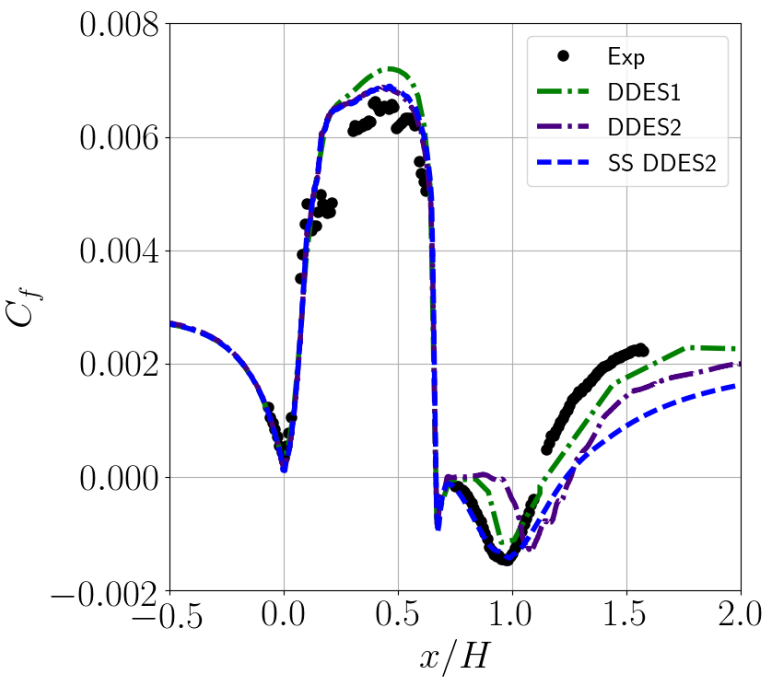

(a) Skin-friction coefficient.

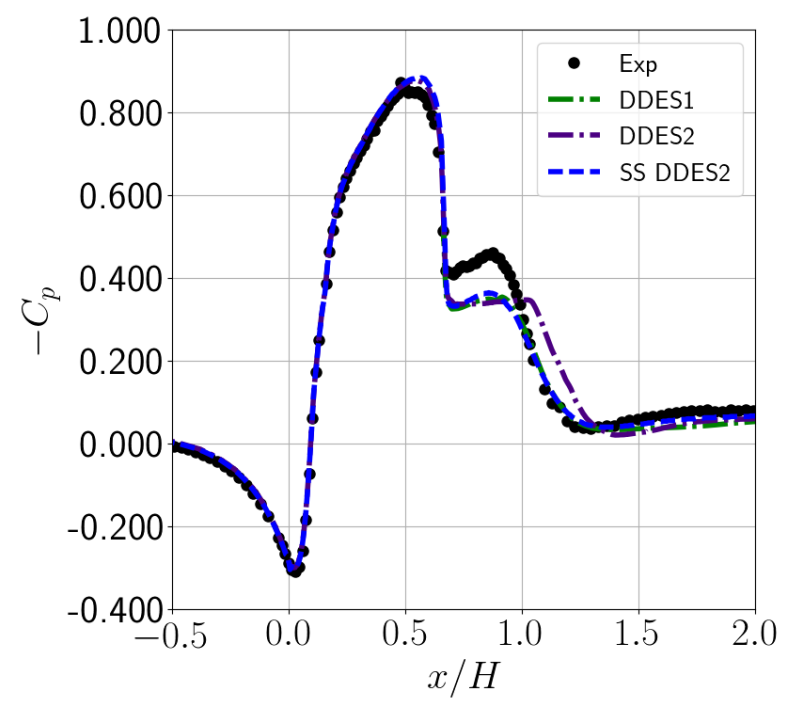

(b) Pressure coefficient.

Fig. 7 Comparison between time-averaged transient results, steady-state results and experimental data along the lower wall.

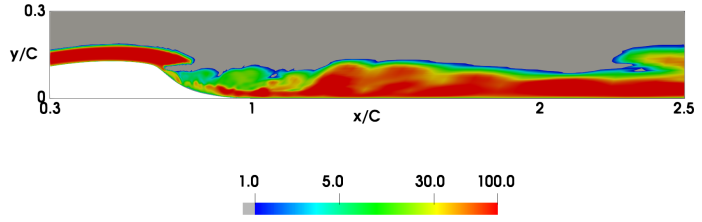

(a) The DDES1 grid.

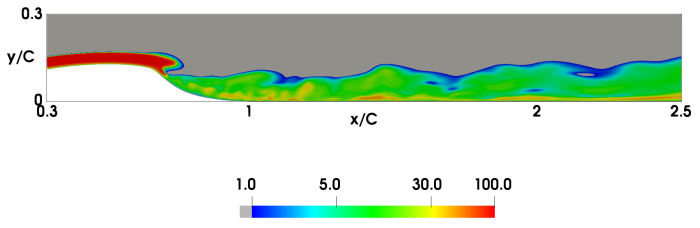

(b) The DDES2 grid.

Fig. 8 Instantaneous eddy viscosity, $v_{t} / v$, at $z / C=z_{\max } / 2$ for the two DDES simulations.

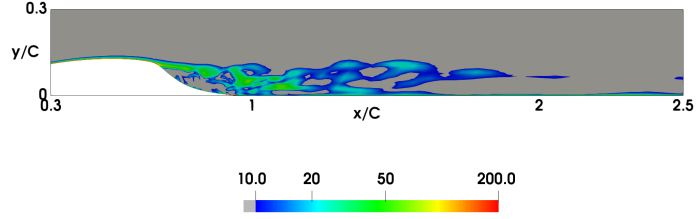

(a) The DDES1 grid.

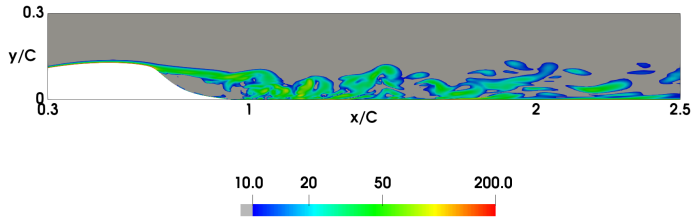

(b) The DDES2 grid.

Fig. 9 Instantaneous vorticity magnitude, $|\Omega| C / U_{\infty}$, at $z / C=z_{\max } / 2$ for the two DDES simulations.

To further visualise the difference in resolved 3D turbulence, an iso-surface of the Q-criterion is presented in Fig. 10 where $Q=1 \cdot 10^{-4}$ is shown and the iso-surface is coloured by stream-wise velocity. Close to the hump there is no substantial visible difference between the two results (the difference in steady shear-layer not visible). However, further downstream, the turbulence obtained using the DDES1 grid decays rapidly, where only very large structures are visible towards the end. This is expected since the grid is too coarse in the that region. The DDES2 grid, on the other hand, can maintain a similar level of turbulence throughout the domain. 


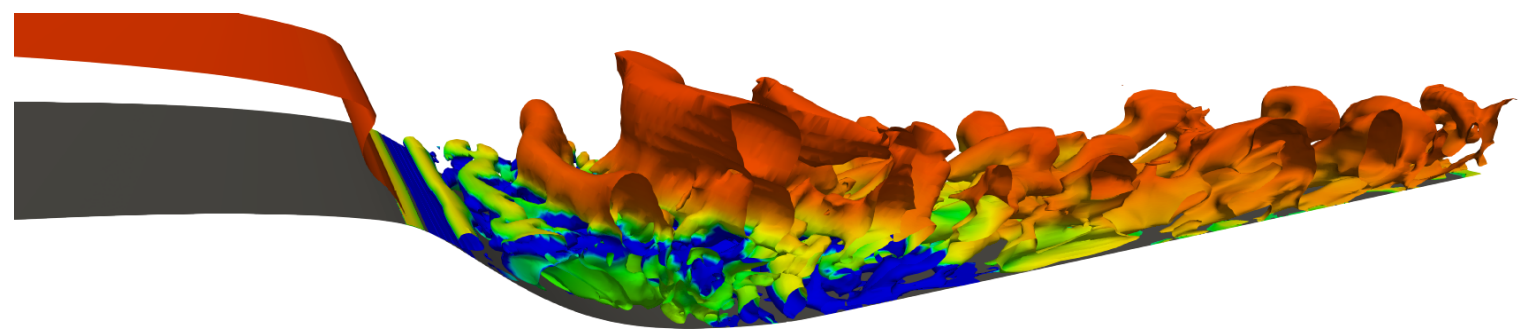

(a) The DDES1 grid.

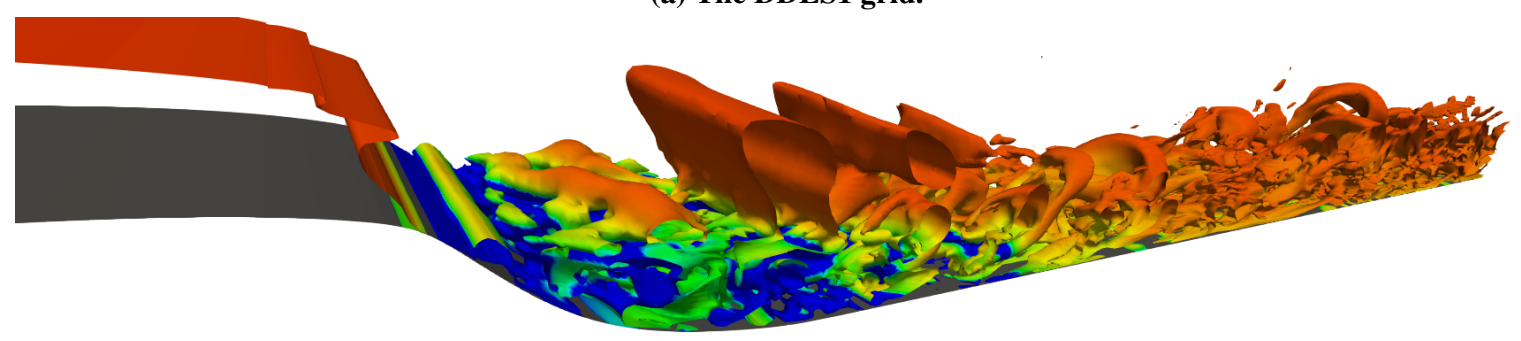

(b) The DDES2 grid.

Fig. 10 Iso-surfaces of the instantaneous $Q$-criterion downstream of the hump, $Q=\frac{1}{4}(|\mathbf{S}|-|\boldsymbol{\Omega}|) /|\boldsymbol{\Omega}|=1 \cdot 10^{-4}$. The iso-surfaces are coloured with stream-wise velocity.

In Fig. 11 the time-averaged velocity profiles at seven different stream-wise locations, obtained from the two transient simulations and the experiments, are compared. The results from the DDES1 grid are presented in Fig. 11a. If considering the RANS results in Fig. 6 the DDES1 result is performing worse in replicating the experimental data for the velocity profiles at $x / C=0.8$ and 0.9 . The large difference for the DDES1 results is suspected to be the result of the late transition from RANS to LES, inside the LES region. There is however a better agreement between the DDES1 results and the experimental data for the velocity profiles further downstream. This emphasises the fact that the DDES1 results predict an earlier re-attachment location, compared to the RANS simulations. The results from the DDES2 grid are not able to capture the flow-behaviour downstream of the hump. The delayed transition and the late re-attachment location result in a significant difference between the DDES2 results on one hand and the experimental data, the RANS simulations and the DDES1 results on the other.

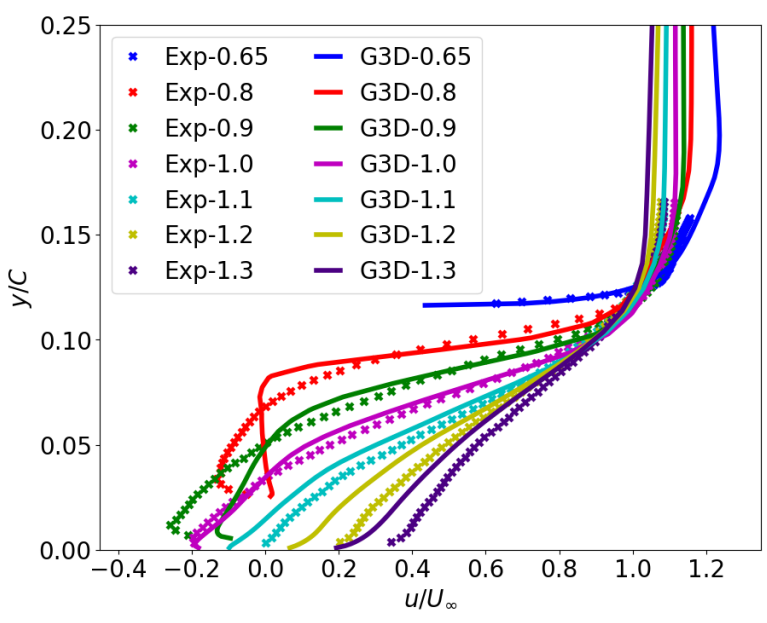

(a) The DDES1 grid.

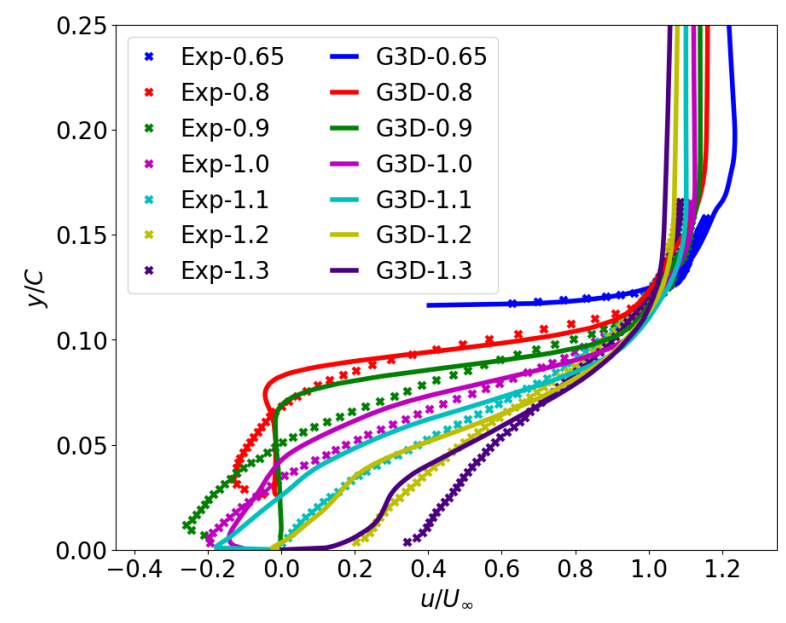

(b) The DDES2 grid.

Fig. 11 Time-averaged velocity profiles downstream of the hump at $x / C=\{0.65,0.8,0.9,1.0,1.1,1.2,1.3\}$. 
The DDES model simulations were done using a dual-time stepping solver. The time-step required by the explicit solver, considering the DDES2 grid, was $\Delta t_{\text {explicit }} \approx 2 \cdot 10^{-8} \mathrm{~s}$, whereas the physical time in the simulations was $\Delta t_{\mathrm{DT}} \approx 5 \cdot 10^{-5} s$ resulting in a speed-up, with 50 inner iterations,

$$
\text { Speed-up }=\frac{\Delta t_{\mathrm{DT}}}{\Delta t_{\text {explicit }} \cdot \text { Number of inner iterations }}=50
$$

The speed-up can be further increased by lowering the maximum number of inner iterations, where an optimum value exists as a compromise between fully converged inner iteration process and computational cost. A test was performed where only 30 inner iterations were used in the dual-time stepping solver. The result obtained were close to identical to theses obtained using 50 inner iterations.

\section{Conclusion}

A well-known test case, often referred to as the 2D NASA wall-mounted hump, is simulated using the in-house CFD solver, G3D::Flow. First, a grid-dependency study is conducted using RANS simulations, where the implementation of the SA turbulence model is verified and a strategy for the grid topology developed. Secondly, transient simulations are performed on two sets of grids, using the DDES turbulence model with an adaptive shear-layer sub-grid length-scale. The solver is an explicit solver using the dual-time stepping method for the transient simulations to allow for time-steps based on flow physics rather than numerical stability. The verification of the SA turbulence model is successful, where a good agreement between the results obtained using G3D::Flow and previously published results obtained using the solver CFL3D is achieved. Furthermore, G3D::Flow is capable of reproducing the experimental data, with the exception being the re-attachment location downstream of the hump. For the transient simulations, the G3D::Flow solver is not capable of reproducing the experimental results for the separated region. This is suspected to be caused by the late transition to resolved 3D turbulence, i.e. transition from RANS to LES. Since different grid topologies are used for the two transient simulations a certain behaviour was noticed. The DDES1 grid, which had a finer grid in the separation region due to clustering of cells and an exponential growth rate towards the outlet, had a better transition to 3D turbulence. However, the DDES2 grid, which had a more uniform cell distribution from the hump towards the outlet, was capable in sustaining the 3D turbulence throughout the domain. From these results, it can be concluded that to improve the performance of the transient simulations, a grid, which is a blend between those two (and even finer in the separation region) should be used. Furthermore, the effects from the domain size should be investigate, where all transient simulations were performed using $z / C=0.2$.

\section{Acknowledgement}

This research work was funded by the Swedish National Aviation Engineering Research Programme, NFFP with financial support from VINNOVA and the Swedish Defence Material Administration (FMV). All CFD simulations were

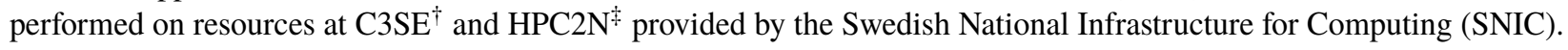

\section{References}

[1] Venkateswaran, S., and Merkle, C. L., "Dual time-stepping and preconditioning for unsteady computations," 33rd Aerospace Sciences Meeting and Exhibit, 1995. doi:10.2514/6.1995-78.

[2] Merkle, C., "Time-accurate unsteady incompressible flow algorithms based on artificial compressibility," 8th Computational Fluid Dynamics Conference, Fluid Dynamics and Co-located Conferences, 1987. doi:10.2514/6.1987-1137.

[3] Siggeirsson, E. M. V., Andersson, N., and Wallin, F., "Numerical and expermiental study on bleed impact on intermediatecompressor duct performance," ASME 2018, Turbo Expo, 2018.

[4] NASA, “2D NASA Wall-Mounted Hump Separated Flow Validation Case (no plenum)," [online], November 2018. Available at: https://turbmodels.larc.nasa.gov/nasahump_val_sa.html[Accessed 22 Nov. 2018].

[5] Spalart, P. R., Deck, S., Shur, M. L., Squires, K. D., Strelets, M. K., and Travin, A., "A new version of detached-eddy simulation, resistant to ambiguous grid densities," Theoretical Computational Fluid Dynamics, Vol. 20, 2006, pp. 181-195. doi:10.1007/s00162-006-0015-0.

\footnotetext{
${ }^{\dagger}$ Chalmers Centre for Computational Science and Engineering

${ }^{\ddagger}$ High Performance Computing Center North
} 
[6] Spalart, P. R., Jou, W.-H., Strelets, M., and Allmaras, S. R., "Comments on the Feasibility of LES for Wings, and on a Hybrid RANS/LES Approach," Proceedings of first AFOR International Conference on DNS/LES, Greyden Press, Ruston, Louisiana, 1997, pp. 137-147. Edited by C. Liu and Z. Liu.

[7] Spalart, P. R., and Allmaras, S. R., "A one-equation turbulence model for aerodynamic flows," 30th Aerospace Sciences Meeting and Exhibit, Aerospace Sciences Meetings, Vol. 1, 1992, pp. 5-21. doi:10.2514/6.1992-439.

[8] Spalart, P. R., Johnson, F. T., and Allmaras, S. R., "Modifications and Clarifications for the Implementation of the SpalartAllmaras Turbulence Model," Seventh International Conference on Computational Fluid Dynamics (ICCFD7), Haway, 2012, pp. 1-11.

[9] Shur, M., Spalart, P. R., Strelets, M., and Travin, A., "Detached-eddy simulation of an airfoil at high angle of attack," Engineering Turbulence Modelling and Experiments 4, Vol. 4, 1999, pp. 669-678. doi:10.1016/B978-008043328-8/50064-3.

[10] Ashton, N., West, A., and Mendonça, F., "Flow Dynamics Past a 30P30N Three-Element Airfoil Using Improved Delayed Detached-Eddy Simulation,” AIAA Journal, Vol. 54, No. 11, 2016, pp. 3657-3667. doi:doi:10.2514/1.J054521.

[11] Probst, A., Radespiel, R., Wolf, C., Knopp, T., and Schwamborn, D., "A Comparison of Detached-eddy Simulation and Reynolds-stress Modelling Applied to the Flow over a Backward-facing Step and an Airfoil at Stall," 48th AIAA Aerospace Sciences Meeting Including the New Horizons Forum and Aerospace Exposition, Aerospace Sciences Meetings, AIAA, 2010, pp. 1-18. doi:doi:10.2514/6.2010-920.

[12] Siggeirsson, E. M. V., Andersson, N., and Wallin, F., "Sensitivity study of the SA-DDES shielding function," 2018 AIAA Aerospace Sciences Meeting, Kissimmee, Florida, 2018.

[13] Eriksson, L.-E., "Development and Validation of Highly Modular Flow Solver Versions in G2DFLOW and G3DFLOW," Tech. Rep. 9970-1162, Volvo Aero Corporation, 1995. Volvo Aero Corporation.

[14] Mårtensson, H., Eriksson, L.-E., and Albråten, P., "Numerical simulations of unsteady wakeflow," The 10th ISABE meeting, Nottingham, United Kingdom, 1991.

[15] Davidson, L., and Peng, S.-H., "Embedded LES Using PANS," 6th AIAA Theoretical Fluid Mechanics Conference, Fluid Dynamics and Co-located Conferences, 2011. doi:10.2514/6.2011-3108.

[16] Menter, F., "Best Practice: Scale-Resolving Simulations in ANSYS CFD,” Tech. Rep. 2, ANSYS Germany GmbH, November 2015.

[17] Andersson, N., Eriksson, L.-E., and Davidson, L., "Large-Eddy Simulation of Subsonic Turbulent Jets and Their Radiated Sound," AIAA Journal, Vol. 43, No. 9, 2005, pp. 1899-1912.

[18] Shur, M. L., Spalart, P. R., Strelets, M. K., and Travin, A. K., "An Enhanced Version of DES with Rapid Transition from RANS to LES in Separated Flows," Flow, Turbulence and Combustion, Vol. 95, No. 4, 2015, pp. 709-737. doi: 10.1007/s10494-015-9618-0.

[19] Guseva, E. K., Garbaruk, A. V., and Strelets, M. K., "Application of DDES and IDDES with shear layer adapted subgrid length-scale to separated flows," Journal of Physics: Conference Series, Vol. 769, 2016.

[20] Vatsa, V. N., Lockard, D. P., and Spalart, P. R., "Grid Sensitivity of SA-Based Delayed-Detached-Eddy-Simulation Model for Blunt-Body Flows,” AIAA Journal, Vol. 55, No. 8, 2017, pp. 2842-2847. doi:10.2514/1.J055685.

[21] Erikson, L., "A preconditioned Navier-Stokes for low Mach number flows," Computational fluid dynamics, Vol. 199, 1996, John Wiley, Chichester (1996).

[22] Turkel, E., "Preconditioning techniques in computational fluid dynamics," Annual Review of Fluid Mechanics, Vol. 31, No. 1, 1999, pp. 385-416. doi:10.1146/annurev.fluid.31.1.385, URL https://doi.org/10.1146/annurev.fluid.31.1.385

[23] Jameson, A., and Baker, T., "Solution of the Euler equations for complex configurations," 6th Computational Fluid Dynamics Conference Danvers. Danvers,MA,U.S.A.., 1983.

[24] Siggeirsson, E. M. V., "Integrated Duct Aerodynamics,” Licentiate Thesis, Chalmers University of Technology, 2018.

[25] Greenblatt, D., Paschal, K. B., Yao, C. ., Harris, J., Schaeffler, N. W., and Washburn, A. E., "A separation control CFD validation test case part 1: Baseline \& steady suction," 2nd AIAA Flow Control Conference, 2004. 